PROCEEDINGS OF THE

AMERICAN MATHEMATICAL SOCIETY

Volume 132, Number 1, Pages 127-133

S 0002-9939(03)06992-2

Article electronically published on March 25, 2003

\title{
A NEW CHARACTERIZATION OF THE UNIT BALL OF $H^{2}$
}

\author{
R. A. KORTRAM \\ (Communicated by Juha M. Heinonen)
}

\begin{abstract}
We derive a new expression for the norm of $H^{2}$ functions; we present some well-known results in a different setting.
\end{abstract}

\section{INTRODUCTION}

In 1915, Pick 3] proved the following result.

Theorem 1. Let $g$ be an analytic function on the unit disc $\Delta$ in the complex plane. Then $|g(z)| \leq 1$ for all $z \in \Delta$ if and only if for all $n \in \mathbb{N}$, for all sequences $z_{1}, z_{2}, \ldots, z_{n}$ in $\Delta$ and for all sequences $\lambda_{1}, \lambda_{2}, \ldots, \lambda_{n}$ we have

$$
\sum_{k=1}^{n} \sum_{l=1}^{n} \frac{1-g\left(z_{k}\right) \overline{g\left(z_{l}\right)}}{1-z_{k} \overline{z_{l}}} \lambda_{k} \overline{\lambda_{l}} \geq 0 .
$$

Ahlfors [1], page 3, gives an elegant proof of this characterization of the unit ball of $H^{\infty}$

In this note we shall present a characterization of the unit ball of $H^{2}$. Our main tool will be an explicit solution of the "minimal interpolation problem" for $H^{2}$ (see [2], page 141). As a byproduct we obtain a new proof of Pick's theorem.

\section{DESCRIPTION OF THE MAIN RESULT}

Let $z_{1}, z_{2}, \ldots, z_{n}$ be a sequence in $\Delta$, and let $b$ be the Blaschke product generated by the sequence

$$
b(z)=\prod_{j=1}^{n} \frac{z-z_{j}}{1-\bar{z}_{j} z} .
$$

We shall prove that the following conditions are equivalent for continuous functions $f$ on $\Delta$ :

1) $f$ lies in the unit ball of $H^{2}$.

2) For every $n \in \mathbb{N}$ and for every sequence $z_{1}, z_{2}, \ldots, z_{n}$ of mutually distinct points in $\Delta$ we have

$$
\sum_{k=1}^{n} \sum_{l=1}^{n} \frac{f\left(z_{k}\right) \overline{f\left(z_{l}\right)}}{1-z_{k} \bar{z}_{l}} \cdot \frac{1}{b^{\prime}\left(z_{k}\right) \overline{b^{\prime}\left(z_{l}\right)}} \leq 1
$$

Received by the editors August 13, 2002.

2000 Mathematics Subject Classification. Primary 30D55.

(C)2003 American Mathematical Society 


\section{PRELIMINARIES}

For mutually distinct points $z_{1}, z_{2}, \ldots, z_{n}$ in $\Delta$ and for $w_{1}, w_{2}, \ldots, w_{n}$ in $\mathbb{C}$ we define

$$
\Lambda=\left\{f \in H^{2}: f\left(z_{j}\right)=w_{j}, j=1,2, \ldots, n\right\} .
$$

$\Lambda$ is not empty; it contains the Lagrange interpolation polynomial

$$
\lambda(z)=l(z) \sum_{k=1}^{n} \frac{w_{k}}{\left(z-z_{k}\right) l^{\prime}\left(z_{k}\right)},
$$

where $l(z)=\prod_{j=1}^{n}\left(z-z_{j}\right)$.

In the context of $H^{p}$ spaces it is more natural to work with the Blaschke interpolation function

$$
\beta(z)=b(z) \sum_{k=1}^{n} \frac{1-\bar{z}_{k} z}{z-z_{k}} \cdot \frac{w_{k}}{b^{\prime}\left(z_{k}\right)\left(1-\left|z_{k}\right|^{2}\right)},
$$

with $b(z)$ defined as in (2). Of course $\beta \in \Lambda$. However, for our purposes we are better off with

$$
\varphi(z)=b(z) \sum_{k=1}^{n} \frac{w_{k}}{\left(z-z_{k}\right) b^{\prime}\left(z_{k}\right)} .
$$

$\varphi \in \Lambda$, and $\varphi$ is analytic on some neighbourhood of $\bar{\Delta}$. $\Lambda$ is a hyperplane in $H^{2}$. With $\varphi$ and $b$ defined as in (4) and (2) we have

$$
\Lambda=\left\{\varphi+b g ; g \in H^{2}\right\} .
$$

Theorem 2. $\varphi$ is the unique solution of the "minimal interpolation problem", i.e., for every $f \in \Lambda \backslash\{\varphi\}$ we have $\|f\|_{2}>\|\varphi\|_{2}$.

Proof. It suffices to show that $\varphi \perp(f-\varphi)$ for every $f \in \Lambda$ (since under those circumstances $\|f\|^{2}=\|\varphi\|^{2}+\|f-\varphi\|^{2}$ ).

From the decomposition $f=\varphi+b g$ we have

$$
\begin{aligned}
\langle f-\varphi, \varphi\rangle & =\langle b g, \varphi\rangle=\frac{1}{2 \pi} \int_{0}^{2 \pi} b\left(e^{i t}\right) g\left(e^{i t}\right) \overline{\varphi\left(e^{i t}\right)} d t \\
& =\frac{1}{2 \pi} \int_{0}^{2 \pi} b\left(e^{i t}\right) g\left(e^{i t}\right) \overline{b\left(e^{i t}\right)} \sum_{k=1}^{n} \frac{\bar{w}_{k}}{\left(e^{-i t}-\bar{z}_{k}\right) \overline{b^{\prime}\left(z_{k}\right)}} d t .
\end{aligned}
$$

Note that $\left|b\left(e^{i t}\right)\right|^{2}=1$. Thus,

$$
\begin{aligned}
& \langle f-\varphi, \varphi\rangle=\sum_{k=1}^{n} \frac{\bar{w}_{k}}{2 \pi \overline{b^{\prime}\left(z_{k}\right)}} \int_{0}^{2 \pi} g\left(e^{i t}\right) \frac{e^{i t}}{1-e^{i t} \bar{z}_{k}} d t \\
& =\sum_{k=1}^{n} \frac{\bar{w}_{k}}{\overline{b^{\prime}\left(z_{k}\right)}} \cdot \frac{1}{2 \pi i} \int_{\Gamma} \frac{g(z)}{1-\bar{z}_{k} z} d z=0,
\end{aligned}
$$

because the integrand is analytic on $\Delta$. 
It will be convenient to have an explicit expression for $\|\varphi\|_{2}$ :

$$
\begin{aligned}
\|\varphi\|_{2}^{2} & =\frac{1}{2 \pi} \int_{0}^{2 \pi}\left|\varphi\left(e^{i t}\right)\right|^{2} d t=\frac{1}{2 \pi} \sum_{k=1}^{n} \sum_{l=1}^{n} \frac{w_{k} \bar{w}_{l}}{b^{\prime}\left(z_{k}\right) \bar{b}^{\prime}\left(z_{l}\right)} \int_{0}^{2 \pi} \frac{d t}{\left(e^{i t}-z_{k}\right)\left(e^{-i t}-\bar{z}_{l}\right)} \\
& =\frac{1}{2 \pi i} \sum_{k=1}^{n} \sum_{l=1}^{n} \frac{w_{k} \bar{w}_{l}}{b^{\prime}\left(z_{k}\right) \bar{b}^{\prime}\left(z_{l}\right)} \int_{\Gamma} \frac{d z}{\left(z-z_{k}\right)\left(1-\bar{z}_{l} z\right)} \\
& =\sum_{k=1}^{n} \sum_{l=1}^{n} \frac{w_{k} \bar{w}_{l}}{1-z_{k} \bar{z}_{l}} \frac{1}{b^{\prime}\left(z_{k}\right) \overline{b^{\prime}\left(z_{l}\right)}} .
\end{aligned}
$$

There are, of course, many other expressions for $\|\varphi\|_{2}$.

\section{Theorem 3.}

$$
\|\varphi\|_{2}=\max \left\{\left|\sum_{k=1}^{n} \frac{w_{k} f\left(z_{k}\right)}{b^{\prime}\left(z_{k}\right)}\right|: f \in H^{2},\|f\|_{2} \leq 1\right\} .
$$

Proof.

$$
\sum_{k=1}^{n} \frac{w_{k} f\left(z_{k}\right)}{b^{\prime}\left(z_{k}\right)}=\frac{1}{2 \pi i} \int_{\Gamma} \frac{f(z) \varphi(z)}{b(z)} d z
$$

hence, by Schwarz's inequality we have

$$
\left|\sum_{k=1}^{n} \frac{w_{k} f\left(z_{k}\right)}{b^{\prime}\left(z_{k}\right)}\right| \leq \frac{1}{2 \pi} \int_{0}^{2 \pi}\left|f\left(e^{i t}\right)\right| \cdot\left|\varphi\left(e^{i t}\right)\right| d t \leq\|f\|_{2} \cdot\|\varphi\|_{2} \leq\|\varphi\|_{2} .
$$

Equality holds for the function $f: z \rightarrow \frac{1}{\|\varphi\|_{2}} \sum_{k=1}^{n} \frac{\bar{w}_{k}}{\left(1-\bar{z}_{k} z \bar{b}^{\prime}\left(z_{k}\right)\right.}$.

An immediate result from Theorem 2 is

Corollary. For every sequence $z_{1}, z_{2}, \ldots, z_{n}$ of mutually distinct points of $\Delta$ we have

$$
\sum_{k=1}^{n} \sum_{l=1}^{n} \frac{1}{1-z_{k} \bar{z}_{l}} \cdot \frac{1}{b^{\prime}\left(z_{k}\right) \overline{b^{\prime}\left(z_{l}\right)}} \leq 1 .
$$

Proof. Take $w_{1}=w_{2}=\ldots=w_{n}=1$. Then $1 \in \Lambda$ and since

$$
\|1\|_{2}=1 \text {, }
$$

we have

$$
1 \geq\|\varphi\|_{2}^{2}=\sum_{k=1}^{n} \sum_{l=1}^{n} \frac{1}{1-z_{k} \bar{z}_{l}} \cdot \frac{1}{b^{\prime}\left(z_{k}\right) \overline{b^{\prime}\left(z_{l}\right)}} .
$$

The equality sign certainly occurs if $0 \in\left\{z_{1}, z_{2}, \ldots, z_{n}\right\}$ :

$$
1=\varphi(0)^{2} \leq \frac{1}{2 \pi} \int_{0}^{2 \pi}\left|\varphi\left(e^{i t}\right)\right|^{2} d t=\|\varphi\|_{2}^{2}=\sum_{k=1}^{n} \sum_{l=1}^{n} \frac{1}{1-z_{k} \bar{z}_{l}} \cdot \frac{1}{b^{\prime}\left(z_{k}\right) \overline{b^{\prime}\left(z_{l}\right)}} .
$$

If $0 \notin\left\{z_{1}, z_{2}, \ldots, z_{n}\right\}$, there is strict inequality.

Because of the uniqueness of $\varphi$ there can be equality only if

$$
b(z) \sum_{k=1}^{n} \frac{1}{\left(z-z_{k}\right) b^{\prime}\left(z_{k}\right)}=1 \text {. }
$$


In this identity for rational functions we let $z \rightarrow \infty$. Since $z_{j} \neq 0, \lim _{z \rightarrow \infty} b(z)$ has a finite value. Therefore, the left-hand side has limit zero.

Remark. The corollary shows that a function satisfying (1) also satisfies (3).

The fact that $\varphi \in \Lambda$ has an interesting reformulation. We start with a lemma.

Lemma 1. The partial fraction decomposition of $\varphi$ is

$$
\varphi(z)=\sum_{k=1}^{n} \sum_{l=1}^{n} \frac{w_{k}}{\left(1-\bar{z}_{l} z\right)\left(1-\bar{z}_{l} z_{k}\right) b^{\prime}\left(z_{k}\right) \overline{b^{\prime}\left(z_{l}\right)}} .
$$

Proof. An elegant way to prove this is to compute both sides of the following identity.

For $z \in \Delta$ we have

$$
\frac{1}{2 \pi i} \int_{\Gamma} \frac{\varphi(\zeta)}{1-\bar{\zeta} z} \cdot \frac{d \zeta}{\zeta}=\overline{\frac{1}{2 \pi i} \int_{\Gamma} \frac{\overline{\varphi(\zeta)}}{1-\zeta \bar{z}} \cdot \frac{d \zeta}{\zeta}}
$$

The left-hand side is equal to

$$
\frac{1}{2 \pi i} \int_{\Gamma} \frac{\varphi(\zeta)}{\zeta-z} d z=\varphi(z)
$$

while the right-hand side is equal to the complex conjugate of

$$
\frac{1}{2 \pi i} \int_{\Gamma} \overline{b(\zeta)} \sum_{k=1}^{n} \frac{\bar{w}_{k}}{\left(\overline{\left.\zeta-z_{k}\right) b^{\prime}\left(z_{k}\right)}\right.} \cdot \frac{1}{1-\zeta \bar{z}} \cdot \frac{d \zeta}{\zeta}
$$

i.e., to the complex conjugate of

$$
\frac{1}{2 \pi i} \int_{\Gamma} \frac{1}{b(\zeta)} \sum_{k=1}^{n} \frac{\bar{w}_{k}}{\left(1-\bar{z}_{k} \zeta\right) \overline{b^{\prime}\left(z_{k}\right)}} \frac{d \zeta}{1-\bar{z} \zeta}
$$

Calculation of the residues at the points $z_{1}, z_{2}, \ldots, z_{n}$ lead to (5).

The condition $\varphi \in \Lambda$ implies that $\varphi\left(z_{j}\right)=w_{j}, j=1, \ldots, n$, i.e.,

$$
\sum_{k=1}^{n} \sum_{l=1}^{n} \frac{w_{k}}{\left(1-\bar{z}_{l} z_{j}\right)\left(1-\bar{z}_{l} z_{k}\right) b^{\prime}\left(z_{k}\right) \overline{b^{\prime}\left(z_{l}\right)}}=w_{j} .
$$

This is equivalent to the assertion that the matrices

$$
B=\left(\beta_{l k}\right)
$$

and its conjugate $\bar{B}=\left(\bar{\beta}_{l k}\right)$ where

$$
\beta_{l k}=\frac{1}{\left(1-\bar{z}_{l} z_{k}\right) b^{\prime}\left(z_{k}\right)}
$$

are inverses of each other, i.e., $B$ and $\bar{B}$ are unitary. 
Proof OF THE MAIN RESUlT

Theorem 4. Let $f$ be a continuous function on the unit disc in the complex plane. Then the following conditions are equivalent:

1. $f$ is analytic and $f$ lies in the unit ball of $H^{2}$.

2. For every $n \in \mathbb{N}$ and for every sequence $z_{1}, z_{2}, \ldots, z_{n}$ of mutually distinct points in $\Delta$ we have

$$
\sum_{k=1}^{n} \sum_{l=1}^{n} \frac{f\left(z_{k}\right) \overline{f\left(z_{l}\right)}}{1-z_{k} \bar{z}_{l}} \cdot \frac{1}{b^{\prime}\left(z_{k}\right) \overline{b^{\prime}\left(z_{l}\right)}} \leq 1
$$

Proof. We split up the proof into two lemmas.

Lemma 2. Let $f$ belong to the unit ball of $H^{2}$, and let a sequence of mutually distinct points $z_{1}, z_{2}, \ldots, z_{n}$ in $\Delta$ be given. Then (3) holds.

Proof. Define $w_{j}=f\left(z_{j}\right) . f$ lies in the hyperplane $\Lambda$ and the element $\varphi$ of $\Lambda$ with minimal norm satisfies

$$
\|\varphi\|_{2} \leq\|f\|_{2} \leq 1 \text {. }
$$

Use of the explicit expression for $\|\varphi\|_{2}$ leads to (3).

Lemma 3. Let $f$ be continuous and assume that $f$ satisfies (3). Then $f$ is analytic and $f$ lies in the unit ball of $H^{2}$.

Proof. We apply (3) for the case $n=1$; an easy computation shows that

$$
|f(z)| \leq \frac{1}{\sqrt{1-|z|^{2}}}
$$

for every choice of $z \in \Delta$.

Let $0<r<\rho<1$, and let $z_{1}, z_{2}, z_{3}, \ldots$ be an enumeration of the rational points of $\bar{\Delta}_{\rho}$. For every $n$ there is a function $\varphi_{n}$ with

$$
\varphi_{n}\left(z_{j}\right)=f\left(z_{j}\right), \quad j=1,2, \ldots, n,
$$

and

$$
\left\|\varphi_{n}\right\|_{2}^{2}=\sum_{k=1}^{n} \sum_{l=1}^{n} \frac{f\left(z_{k}\right) \overline{f\left(z_{l}\right)}}{1-z_{k} \bar{z}_{l}} \cdot \frac{1}{b^{\prime}\left(z_{k}\right) \overline{b^{\prime}\left(z_{l}\right)}} \leq 1 .
$$

Thus, $\varphi_{n}$ lies in the unit ball of $H^{2}$, and so by Lemma2, we have for every sequence $\zeta_{1}, \zeta_{2}, \ldots, \zeta_{n}$ in $\Delta$

$$
\sum_{k=1}^{m} \sum_{l=1}^{m} \frac{\varphi_{n}\left(\zeta_{k}\right) \overline{\varphi_{n}\left(\zeta_{l}\right)}}{1-\zeta_{k} \bar{\zeta}_{l}} \cdot \frac{1}{b^{\prime}\left(z_{k}\right) \overline{b^{\prime}\left(z_{l}\right)}} \leq 1
$$

It follows from (6) that

$$
\left|\varphi_{n}(\zeta)\right| \leq \frac{1}{\sqrt{1-|\zeta|^{2}}}
$$

hence the sequence $\varphi_{1}, \varphi_{2}, \ldots$ is uniformly bounded on $\bar{\Delta}_{\rho}$. Therefore, it contains a locally uniformly convergent subsequence $\varphi_{n_{j}}$. At the points $z_{1}, z_{2}, \ldots$ the subsequence converges to $f$. By the continuity of $f$ and the fact that $\left\{z_{1}, z_{2}, \ldots\right\}$ is dense in $\Delta_{\rho}$ we see that

$$
\lim _{n_{j} \rightarrow \infty} \varphi_{n_{j}}=f .
$$


This shows that $f$ is analytic on $\Delta_{\rho}$ for all $\rho<1$. Because of uniform convergence on $\Gamma_{r}$, we have

$$
\frac{1}{2 \pi} \int_{0}^{2 \pi}\left|f\left(r e^{i t}\right)\right|^{2} d t=\lim _{n_{j} \rightarrow \infty} \frac{1}{2 \pi} \int_{0}^{2 \pi}\left|\varphi_{n_{j}}\left(r e^{i t}\right)\right|^{2} d t \leq 1 .
$$

Thus, $f \in H^{2}$ and $\|f\|_{2} \leq 1$.

Lemma 2 and Lemma 3 together constitute a proof of the theorem.

Corollary. For $f \in H^{2}$ we define

$$
\begin{aligned}
\nu(f)=\sup \left\{\sum_{k=1}^{n} \sum_{l=1}^{n} \frac{f\left(z_{k}\right) \overline{f\left(z_{l}\right)}}{1-z_{k} \bar{z}_{l}}\right. & \cdot \frac{1}{b^{\prime}\left(z_{k}\right) \overline{b^{\prime}\left(z_{l}\right)}} ; \\
& \left.z_{1}, z_{2}, \ldots, z_{n} \text { mutually distinct points of } \Delta\right\} .
\end{aligned}
$$

Then $\nu(f)=\|f\|_{2}^{2}$.

Proof. Assume that $\nu(f)=1$. Then by Lemma $3\|f\|_{2}^{2} \leq 1$. If $\|f\|_{2}^{2}<\lambda^{2}<1$ for some $\lambda$, then we have $\left\|\frac{1}{\lambda} f\right\|<1$ but $\nu\left(\frac{1}{\lambda} f\right)>1$ which is impossible by Lemma 2 ,

In a similar way we can show that $\|f\|_{2}^{2}=1$ implies that $\nu(f)=1$. By the homogeneity of $\nu$ and $\|\quad\|_{2}^{2}$ it follows that for all $f \in H^{2}: \nu(f)=\|f\|_{2}^{2}$.

\section{PICK'S THEOREM}

As an application of our results we shall give a proof of Pick's theorem.

Let $g$ belong to the unit ball of $H^{\infty}$, and let $z_{1}, z_{2}, \ldots, z_{n}$ be a sequence of mutually distinct points in $\Delta$. Let $w_{1}, w_{2}, \ldots, w_{n}$ be an arbitrary sequence of complex numbers. We consider the hyperplanes $\Lambda$ and $\Lambda_{g}$ where

$$
\Lambda_{g}=\left\{f \in H^{2}: f\left(z_{j}\right)=w_{j} g\left(z_{j}\right), j=1,2, \ldots, n\right\} .
$$

Of course, if $f \in \Delta$, then $g \cdot f \in \Delta_{g}$, and by Theorem 2 applied to $\Lambda_{g}$ we have

$$
\|g f\|_{2}^{2} \geq \sum_{k=1}^{n} \sum_{l=1}^{n} \frac{w_{k} g\left(z_{k}\right) \overline{w_{l} g\left(z_{l}\right)}}{1-z_{k} \bar{z}_{l}} \frac{1}{b^{\prime}\left(z_{k}\right) \overline{b^{\prime}\left(z_{l}\right)}} .
$$

Let $\varphi$ be, as before, the element of $\Lambda$ with smallest norm. From $\|g\|_{\infty} \leq 1$ we obtain

$$
\|g \varphi\|_{2} \leq\|\varphi\|_{2} .
$$

Combining these steps leads to

$$
\begin{aligned}
& \sum_{k=1}^{n} \sum_{l=1}^{n} \frac{w_{k} \bar{w}_{l}}{1-z_{k} \bar{z}_{l}} \cdot \frac{1}{b^{\prime}\left(z_{k}\right) \overline{b^{\prime}\left(z_{l}\right)}} \\
& \quad=\|\varphi\|_{2}^{2} \geq\|g \varphi\|_{2}^{2} \geq \sum_{k=1}^{n} \sum_{l=1}^{n} \frac{w_{k} \bar{w}_{l} g\left(z_{k}\right) \overline{g\left(z_{l}\right)}}{1-z_{k} \bar{z}_{l}} \cdot \frac{1}{b^{\prime}\left(z_{k}\right) \overline{b^{\prime}\left(z_{l}\right)}},
\end{aligned}
$$

i.e., to

$$
\sum_{k=1}^{n} \sum_{l=1}^{n} \frac{1-g\left(z_{k}\right) \overline{g\left(z_{l}\right)}}{1-z_{k} \bar{z}_{l}} \cdot \frac{w_{k} \bar{w}_{l}}{b^{\prime}\left(z_{k}\right) \overline{b^{\prime}\left(z_{l}\right)}} \geq 0
$$


and since the sequence $w_{1}, w_{2}, \ldots, w_{n}$ is arbitrary, we have for all choices of $\lambda_{1}$, $\lambda_{2}, \ldots, \lambda_{n}$

$$
\sum_{k=1}^{n} \sum_{l=1}^{n} \frac{1-g\left(z_{k}\right) \overline{g\left(z_{l}\right)}}{1-z_{k} \bar{z}_{l}} \cdot \lambda_{k} \bar{\lambda}_{l} \geq 0 .
$$

By the choice $n=1, \lambda_{1}=1$ we see that the converse is trivial.

\section{REFERENCES}

1. Lars V. Ahlfors, Conformal Invariants, McGraw Hill (New York), 1973. MR 50:10211

2. P.L. Duren, Theory of $H^{p}$ spaces, Academic Press (New York, London), 1970. MR 42:3552

3. G. Pick; Über die Beschränkungen analytischer Funktionen, welche durch vorgeschriebene Werte bewirkt werden, Math. Ann. 77 (1915), 7-23.

Department of Mathematics, Catholic University, Toernooiveld, 6525 ED Nijmegen, THE NETHERLANDS

E-mail address: kortram@math.kun.nl 